\title{
An Examination of Pre-Service Teachers' Competencies in Lesson Planning
}

\author{
Serhat Süral \\ Correspondence: Serhat Süral, Pamukkale University, Faculty of Education, Department of Curriculum and Instruction, \\ Denizli Turkey.
}

Received: December 24, 2018

Accepted: January 8, 2019

Online Published: January 18, 2019

doi:10.11114/jets.v7i3.3902

URL: https://doi.org/10.11114/jets.v7i3.3902

\begin{abstract}
This study concerns the investigation of pre-service teachers' competencies in lesson planning from different perspectives. In this respect, it is intended to develop a roadmap to figure out the pre-service teachers' competency levels in lesson planning pertaining to a four-year- education program. The study was designed as a quantitative study and the general screening model was used. This model is designed with the relational screening model. The study population comprises of $3^{\text {rd }}$ grade and senior students majoring in classroom, preschool, science, social sciences, mathematics and Turkish language teaching departments at Pamukkale University in the 2018-2019 academic year. "The Competency Scale for Lesson Planning" developed by the researcher attempted to determine pre-service teachers' competencies in lesson planning. Considering reliability and validity levels of the scale, this scale can also be administered to different sample groups. The results denoted that pre-service teachers have an optimal level of competency in lesson planning.
\end{abstract}

Keywords: lesson plan, competency, factor analysis, theoretical competency, practical competency

\section{Introduction}

Plan is a template that consists of diagrams or steps used to achieve a goal. More specifically, it is a draft which provides a framework about what, when, why and how to teach or a written document that explains teachers' course activities. The fundamental objective of the instructional planning is to enhance the quality of the instruction and the impact of the instructional program in practice. Planning enables teachers to systematically organize various learning tools and use them regularly. The instruction program helps teachers monitor their own teaching activities and identify how they affect the teaching process. In other words, it supports the reflective teacher role. Plan can be employed as a method to identify which instructional activities will be chosen and why and how they will be implemented and which supplementary and complimentary resources and tools will be used and how the success gained will be measured. In this respect, all these mentioned objectives are predetermined on a paper through a lesson plan (Demirel and Yağc1, 2003).

As the first stage of the teaching process, lesson planning also determines the next stages of the teaching. To achieve predetermined goals in a set timeline, it is necessary to organize the works to be performed, timetable of activities and the resources to be used (Vural, 2006). In addition to that, preparing a lesson plan is a duty for teachers in terms of professional responsibility and legislation. "Directive on Planned Execution of Education and Training Studies" published in the Journal of the Communique No: 2551 by Ministry of National Education underlines the necessity, benefits and principles of the preparing a lesson planning. Consequently, preparing a lesson plan has officially become mandatory.

Senemoglu (2003) stresses out following three functions of a lesson planning: emotionally boosting learners' self-confidence, organizing instructional elements to be used for learning, enabling instructors to monitor, evaluate and fix their teaching activities, in other words, helping instructors adopt reflective thinking. Thanks to a planned instruction process, teachers will feel self-confident and easily handle unexpected occasions in the classroom environment by behaving calm and easy. Since a lesson plan is composed of various phases, the instruction will be fulfilled systematically, thereby minimizing class management issues. Further, lesson planning activities will help teachers comprehend the student, instruction methods, tools and methods and evaluation concerning the next instruction process.

Bilen (2002) emphasizes the importance of preparing a detailed lesson plan in terms of enhancing the quality of the teaching. In a similar vein, Ercoşkun; Nalçacı; Kılıç (2004) assert that teachers should be encouraged to consider their role, how and why they perform in the education and teaching process so that an effective lesson planning can be ensured. Student-cantered lesson plans guide teachers to conduct an effective educational and instructional activities. According to 
Driscoll and Freiberg (1992), lesson planning makes the instruction more purposeful and effective. Conducting researches on how teachers identify their instructional objectives in lesson planning as well as their decision-making processes and strategy formulation, Zahorik argues that specifying objectives and in-class activities are fundamental parts of an effective instruction. Eventually, an unplanned lesson results in ineffective and purposeless instruction.

Senemoglu (2005) notes that the success of the teaching process depends greatly upon the high quality lesson planning. Lesson planning has basically three functions throughout the instruction process: first, it emotionally boosts learners' self-confidence, second, it organizes instructional elements to be used for learning, third and last, it enables instructors to monitor, evaluate and fix their teaching activities, in other words, help them adopt reflective thinking. Likewise, Tan; Kayabaşı; Erdoğan (2002) highlight that lesson planning boosts teacher performance and provides an appropriate learning environment, thereby fulfilling educational goals and increasing student engagement. Küçïkahmet (2005) stresses out instructional planning encourages teachers to consider what their role are, how and why they perform during education and teaching process so that an effective lesson planning can be ensured.

According to the sixth provisions of Directive of Ministry of National Education on "Planned Execution of Education and Training Studies" published in the Journal of the Communique of MoNE (Ministry of National Education) in 2008, "In educational institutions, teachers need to be aware that they are legally obligated to fulfill lesson preparation. In this respect, lesson preparation is a necessity in terms of education. Education and teaching is a progressive work that needs to be addressed in a rigorous and systematic manner. The teacher should pay a considerable attention to lesson planning and lesson preparation in order to achieve an efficient and effective education-teaching process. Moreover, regulations of preschool education, primary education, secondary education, vocational and technical education and non-formal education institutions and their education-teaching programs emphasize that instructional activities need to be implemented in a planned and systematic way."

A plenty of studies underline the importance of preparing a lesson plan and planning for teachers' classroom performance. Similarly, teaching programs allow pre-service teachers to improve their teaching skills since they will have the opportunity to conduct actual or almost actual teaching practices as well as attending theoretical courses (Beeth \& Adadan, 2006; Goodlad, 1991; Meade 1991; Peker, 2009; Roth \& Tobin, 2001; Sachs, 1997; Sumpter, 1995; Tigchelaar \& Korthagen, 2004).

The significance of the study is to figure out the pre-service teachers' competency levels in lesson planning pertaining to a four-year- education program and correspondingly to develop a roadmap. Also, this study seeks to examine the competency levels of the pre-service teachers studying at education faculties in the 2018-2019 academic year. The present study aims to analyze the competency levels of the pre-service teachers from education faculties in the 2018-2019 academic year in terms of different perspectives. Accordingly, answers to the following questions were sought:

1. What is the level of the factor analysis values pertaining to "The Competency Scale for Lesson Planning"?

2. What is the competency level of the pre-service teachers in lesson planning?

3. Do pre-service teachers' competency levels in lesson planning significantly vary according to gender, department, and grade level variables?

\section{Method}

This section provides methodological aspects of the study. In this sense, the research model, the study population and the sample size, the validity and reliability study of data gathering tools and other tests used for data analysis were presented.

\subsection{Research Model}

The study was designed as a quantitative study and the general screening model was used. This model is designed with the relational survey model. The relational survey models are research models which aim to determine the presence and the level of change variance between two or more variable (Gay, 1987; Gall, J.; Gall, M.D. and Borg, 1999).

\subsection{Study Population and Sample Size}

The study population comprises of $3^{\text {rd }}$ grade and senior students majoring in classroom, preschool, science teaching, and social sciences teaching departments at Pamukkale University in the 2018-2019 academic year. Since the number of students majoring in these mentioned departments is almost equal to each other, these four departments were selected to identify validity and reliability coefficients of the study more appropriately. In addition to that, this study aimed to monitor pre-service teachers' progress in the final two years of the teacher education programs. Accordingly, 3rd grade students were included as the subject of the study. 
Table 1. Frequency Distribution of the Sample Group According to the Identified Variables (Actual Application)

\begin{tabular}{|c|c|c|c|}
\hline Variable & Groups & $\begin{array}{l}\text { Descriptive Data } \\
\mathrm{F}\end{array}$ & $\%$ \\
\hline \multirow{2}{*}{ Gender } & Female & 480 & 77.4 \\
\hline & Male & 140 & 22.6 \\
\hline \multirow{6}{*}{ Department } & Classroom Teaching & 138 & 22.3 \\
\hline & Preschool Teaching & 174 & 28.1 \\
\hline & Mathematics Teaching & 65 & 10.5 \\
\hline & Science Teaching & 100 & 16.2 \\
\hline & Social Sciences Teaching & 70 & 11.2 \\
\hline & Turkish Language Teaching & 73 & 11.7 \\
\hline \multirow{2}{*}{ Grade Level } & $3^{\text {rd }}$ Grade & 375 & 60.4 \\
\hline & Senior & 245 & 39.6 \\
\hline Grand Total & & 620 & 100 \\
\hline
\end{tabular}

Disproportionate stratified sampling method was run during the phase of actual application. The number of population was determined as 2918, whereas the number of sample group was calculated as 339 people (Balc1, 1995, p.111). However, the number of the sample group was increased to reach more reliable results and thus data collection involved the participation of 620 people.

\subsection{Data Collection Tools}

"The Competency Scale for Lesson Planning" utilized in the study was developed by the researcher to identify pre-service teachers' competencies in lesson planning. 20 teachers who have been working in public and private schools for minimum 3 years and maximum 17 years were asked to answer following open-ended questions: "What are your opinions and thoughts on your theoretical knowledge on lesson planning, instructional program elements and the significance of the relation among these elements, the problems that you encounter during the implementation of a lesson plan and the necessity of lesson planning?". In view of the pre-service teachers' answers, potential scale items sentences were discarded. Having performed reliability and validity analysis, the initial 34 item-scale were reduced to 23 item-scale with two sub-dimensions. Measuring competency level of teachers or pre-service teachers in lesson planning, the scale consisted of two sub-dimensions, namely, theoretical competency (item 1, 3, 4, 5, 6, 7, 13, 16, 18, 22 and item 23) and practical competency (item 2, 8, 9, 10,11, 12,14,15, 17, 19, 20 and item 21.

Kline (1994) argues that considering the item number or factor number in the measurement tool of the sample size, the sample size can be 10 times greater than item number during the phase of the scale development process (Cited in: Çokluk, Şekercioğlu \& Büyüköztürk, 2012). Therefore, data were collected through 241 teachers working as classroom $(\mathrm{N}=92)$, preschool $(\mathrm{N}=48), \quad$ science $(\mathrm{N}=30), \quad$ social $(\mathrm{N}=22), \quad$ mathematics $(\mathrm{N}=18)$ and Turkish language $(\mathrm{N}=31)$ teachers at public and private schools in the province of Denizli. Afterwards, reliability analysis of factor analysis and pilot study were performed.

Table 2. Reliability Coefficients of the Scale and its sub-dimensions

\begin{tabular}{lll}
\hline & \multicolumn{2}{c}{ Cronbach Alpha Values } \\
\hline Factors & Actual & Pilot \\
& Application & Application \\
1. Theoretical Competency Sub-dimension & .812 & .778 \\
2. Practical Competency Sub-dimension & .886 & .792 \\
Grand Total & $\mathbf{. 8 6 7}$ & $\mathbf{. 7 8 2}$ \\
\hline
\end{tabular}

It was underlined that a reliability value of 0.60 was required for preliminary studies, 0.80 for fundamental studies and between 0.90 and 0.95 for practical studies. On the other hand, the reliability coefficients values concerning the social sciences differ according to the research type, a reliability value of 0.70 for scientific-based studies is required and studies where ability, skills and interest are needed require a reliability coefficient level of 0.85. (Şencan, 2005).According to the reliability analysis of the pilot study where all items were included, Cronbach Alpha value was found to be .782, whereas the results of the reliability analysis of the actual study indicated a Cronbach Alpha value with .867. We can thus contend that the scale can be used as a reliable measurement tool. On the other hand, given the reliability coefficients of the sub-dimensions, we can generally imply that reliable coefficients were obtained from the sample group.

\subsection{Data Analysis}

Each item in the draft scale was first transformed into computer according to 214 pre-service teachers' responses and both each item and total scores of the pre-service teachers were calculated. Explanatory Factor Analysis (EFA) was utilized for structural validity. Confirmatory factor analysis (CFA) was performed to see the fit indices of the factors identified. The suitability of the data for factor analysis and sample size was determined by running the Kaiser-Meyer-Olkin and Bartlett's Test of Sphericity. The suitability of the data for factor analysis was tested through anti-image correlation matrix. 
The Kolmogorov-Smirnov test was performed to evaluate the normality of the variables. The distribution of the variables identified was evaluated and afterwards it was agreed on which parametric or non-parametric test would be applied. Lastly, alongside descriptive statistics, arithmetic mean and standard deviation was used to identify digital citizenship levels of the teacher candidates.

\section{Findings}

In attempt to seek answers to the sub problems posed in the study, a series of analyses conducted and findings of these analyses were presented in this section.

\subsection{Factor Analysis Values of the Competency Scale for Lesson Planning}

Initially, factor analysis was performed using anti-image correlation matrix. The diagonal of anti-image correlation matrix should be greater than .50 (Can, 2014). Items showing a correlation of less than .50 were removed from the survey. The remaining items were subjected to factor analysis. In light of the anti-image correlation matrix results shown in Table 3 , it is seen that the diagonal values vary between .530 (9th item) and .920 (3rd item).

Table 3. Anti-Image Correlation Matrix

\begin{tabular}{|c|c|c|c|c|c|c|c|c|c|c|c|c|c|c|c|c|c|c|c|c|c|c|c|}
\hline & $\vec{\Xi}$ & $\begin{array}{l}\stackrel{N}{E} \\
\text { E }\end{array}$ & 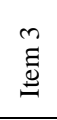 & $\begin{array}{l}\vec{े} \\
\stackrel{\Xi}{\Xi} \\
\end{array}$ & 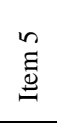 & 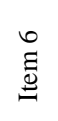 & $\widehat{\bar{E}}$ & $\begin{array}{l}\infty \\
\Xi \\
\Xi\end{array}$ & $\begin{array}{l}\stackrel{\Xi}{\Xi} \\
\stackrel{\Xi}{E}\end{array}$ & $\begin{array}{l}\stackrel{ }{\Xi} \\
\stackrel{\Xi}{\Xi}\end{array}$ & $\begin{array}{l}= \\
\text { 䮍 }\end{array}$ & 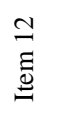 & $\begin{array}{l}\stackrel{m}{\Xi} \\
\stackrel{\Xi}{\underline{E}}\end{array}$ & $\begin{array}{l}\underset{\Xi}{\Xi} \\
\stackrel{\Xi}{\Xi}\end{array}$ & 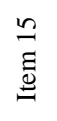 & $\begin{array}{l}\stackrel{0}{\Xi} \\
\stackrel{\Xi}{\Xi}\end{array}$ & 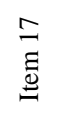 & $\frac{\infty}{\stackrel{\infty}{E}}$ & $\begin{array}{l}\stackrel{\vartheta}{\vec{\Xi}} \\
\stackrel{\Xi}{=}\end{array}$ & 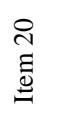 & $\begin{array}{l}\overline{\widetilde{N}} \\
\text { E्ञ } \\
\text { E }\end{array}$ & 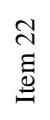 & $\begin{array}{l}\tilde{N} \\
\tilde{\Xi} \\
\tilde{\Xi}\end{array}$ \\
\hline$=$ & స్ర & $\cong$ & તิ & है & $\mathscr{f}$ & ర్లి & $\cong$ & 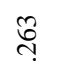 & $\stackrel{\vartheta}{\varrho}$ & $\tilde{\mathcal{F}}$ & $\begin{array}{l}\infty \\
\stackrel{\infty}{o} .\end{array}$ & గి. & $\stackrel{\overbrace{}}{\leftrightarrows}$ & $\tilde{๗}$ & กิ & $\hat{8}$ & $\ddot{\simeq}$ & $\stackrel{\mathfrak{f}}{\mathrm{f}}$ & 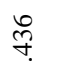 & 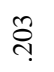 & $\hat{s}$ & $\stackrel{\text { 导 }}{ }$ & $\stackrel{\nearrow}{\simeq}$ \\
\hline$\simeq$ & $\stackrel{\bullet}{?}$ & $\stackrel{n}{?}$ & 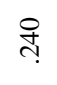 & 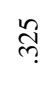 & $\underset{\sim}{\Delta}$ & $\stackrel{\mathscr{a}}{\leftrightarrows}$ & $\stackrel{\infty}{8}$ & $\hat{\hat{o}}$ & $\stackrel{\sim}{\underset{i}{i}}$ & $\frac{\stackrel{n}{n}}{i}$ & $\stackrel{\partial}{\partial}$ & 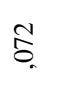 & है. & 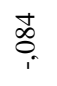 & $\stackrel{?}{\cong}$ & \&̊. & $\stackrel{?}{\text { s. }}$ & $\stackrel{8}{0}$ & $\stackrel{\sigma}{\varrho}$ & $\stackrel{?}{\stackrel{0}{i}}$ & $\frac{\mathfrak{y}}{i}$ & $\stackrel{\infty}{\simeq}$ & ? \\
\hline 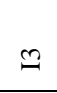 & 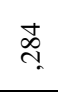 & $\frac{8}{1}$ & హ్ & $\hat{m}$ & $\stackrel{n}{0}$ & 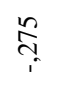 & $\tilde{n}_{i}^{n}$ & స్ & $\exists$ & $\vec{m}$ & 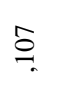 & $\underset{i}{\stackrel{ \pm}{\prime}}$ & $\stackrel{\infty}{+}$ & $\stackrel{\tilde{n}}{i}$ & $\stackrel{\infty}{\circ}$ & $\begin{array}{l}0 \\
\text { ö }\end{array}$ & $\underset{i}{\stackrel{ \pm}{i}}$ & \& & $\stackrel{ \pm}{i}$ & $\frac{g}{i}$ & :্ণ & ठิ & $\tilde{c}_{i}$ \\
\hline \pm & $\stackrel{\infty}{i}$ & ธิ. & $\underset{i}{\stackrel{+}{i}}$ & बे & $\hat{o}_{i}$ & 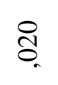 & $\vec{m}$ & 아. & 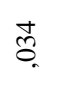 & $\underset{+}{+}$ & $\stackrel{0}{n}$ & $\tilde{o}_{i}$ & $\overrightarrow{\vec{i}}$ & $\vec{\Xi}$ & $\underset{i}{I}$ & సิ & $\vec{\varnothing}$ & $\begin{array}{l}\text { t } \\
\text { Oे } \\
\text { in }\end{array}$ & $\stackrel{\circ}{\sim}$ & $\bar{r}$ & $\stackrel{\overbrace{}}{\stackrel{2}{r}}$ & 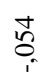 & $\stackrel{\Xi}{\Xi}$ \\
\hline $\mathscr{n}$ & ర్రి & 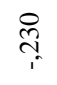 & \&. & $\mathbb{8}_{i}$ & $\stackrel{n}{n}$ & $\frac{\mathrm{O}}{0}$ & $\underset{i}{\stackrel{g}{i}}$ & $\hat{o}_{i}$ & $\stackrel{\text { ڤ్ }}{i}$ & हे. & $\stackrel{\text { ते }}{i}$ & $\stackrel{\Re}{\infty}$ & $\stackrel{\infty}{5}$ & $\stackrel{\tilde{o}_{i}}{{ }_{i}}$ & $\stackrel{8}{0}$ & $\Xi$ & $\ddot{q}_{i}$ & $\hat{\hat{s}}$ & ते & 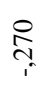 & 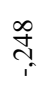 & 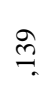 & $\stackrel{\infty}{\underset{1}{\sim}}$ \\
\hline$\varrho$ & $\stackrel{+}{ \pm}$ & $\mathbb{B}_{i}$ & $\mathscr{\delta}_{i}$ & d̦ & $\stackrel{\infty}{i}$ & $\underset{\infty}{\infty}$ & $\bar{s}$ & $\check{0}$ & $\stackrel{m}{=}$ & $\tilde{\delta}$ & $\underset{\text { ণ্ }}{\text { i. }}$ & $\stackrel{8}{\circ}$ & $\underset{\mathrm{O}}{\mathrm{O}}$ & 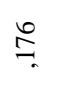 & ঙ্. & $\widetilde{\delta}$ & 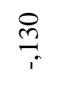 & in & $\stackrel{8}{\circ}$ & $\cong$ & $\Xi$ & $\overline{\mathrm{s}}_{i}$ & 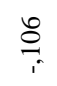 \\
\hline$\simeq$ & $\stackrel{\circ}{\circ}$ & o & $\stackrel{\infty}{\simeq}$ & $\stackrel{M}{\exists}$ & ชิ. & 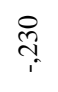 & $\hat{\sigma}$ & $\stackrel{0}{m}$ & $\stackrel{\circ}{\curvearrowright}$ & $\underset{i}{\stackrel{t}{i}}$ & లి. & $\mathscr{8}_{i^{\prime}}$ & $\stackrel{\infty}{\simeq}$ & $\tilde{g}_{i}$ & $\tilde{\vartheta}_{i}$ & 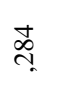 & $\stackrel{8}{:}$ & $\frac{\stackrel{g}{i}}{i}$ & $\bar{z}_{i}$ & $\stackrel{\infty}{\stackrel{\infty}{0}}$ & ठิ & $\underset{i}{ \pm}$ & $\stackrel{0}{0}$ \\
\hline$\stackrel{\infty}{.}$ & : & $\exists$ & $\stackrel{t}{\sigma}$ & $\underset{\sim}{\stackrel{\mathcal{I}}{i}}$ & $\underset{\sim}{+}$ & $\frac{8}{i}$ & $\underset{0}{\stackrel{t}{0}}$ & $\stackrel{\infty}{\curvearrowright}$ & 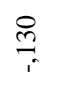 & $\stackrel{n}{0}_{i}$ & $\tilde{\delta}$ & $\overrightarrow{\widetilde{\pi}}$ & $\stackrel{్}{.}$ & ô & $\begin{array}{l}\mathscr{\infty} \\
\stackrel{1}{1} \\
i\end{array}$ & $\begin{array}{l}\hat{\infty} \\
\tilde{o}_{i}\end{array}$ & స్లి & $\stackrel{\vec{\partial}}{\overrightarrow{0}}$ & $\stackrel{巳}{\simeq}$ & $\begin{array}{l}\infty \\
\infty_{0}^{\infty} \\
i^{,}\end{array}$ & 8 & 范 & $\stackrel{\Xi}{0}$ \\
\hline$\Omega$ & $\stackrel{8}{-}$ & $\overline{8}$ & $\overrightarrow{8}$ & $\stackrel{\circ}{0}$ & $\overrightarrow{\tilde{o}}$ & $\underset{i}{i}$ & $\stackrel{\circ}{\curvearrowright}$ & $\stackrel{\infty}{0}$ & 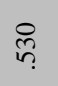 & $\begin{array}{l}\stackrel{0}{0} \\
\stackrel{0}{0} .\end{array}$ & $\bar{\sigma}_{i}$ & $\hat{s}_{i}$ & $\stackrel{\infty}{\hookrightarrow}$ & ટ્రి & $\stackrel{\mathrm{I}}{\simeq}$ & 守 & $\bar{\delta}$ & 茪 & :্ণ & ले & 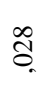 & 8 & $\frac{g}{i}$ \\
\hline$\varrho$ & $\widetilde{\widetilde{\imath}}$ & 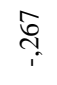 & $\stackrel{\infty}{\stackrel{0}{.}}$ & $\stackrel{\tilde{2}}{i}$ & $\bar{\delta}$ & $\stackrel{\infty}{=}$ & $\underset{i^{\prime}}{\stackrel{\infty}{5}}$ & $\underset{\mathrm{I}}{\mathrm{i}}$ & $\underset{i}{\stackrel{ \pm}{*}}$ & $\stackrel{\leftrightarrow}{a}$ & $\stackrel{\infty}{\sim}$ & $\stackrel{\infty}{\stackrel{2}{i}}$ & $\underset{m}{\vec{f}}$ & $\stackrel{m}{\vec{c}}$ & $\underset{i}{\stackrel{+}{\sim}}$ & \&. & $\underset{d}{t}$ & 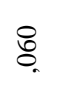 & $\tilde{\delta}$ & $\stackrel{0}{m}$ & है. & $\stackrel{8}{\stackrel{8}{0}}$ & స్తి \\
\hline$\Xi$ & $\underset{+}{ \pm}$ & ڤે & $\stackrel{8}{1}$ & $\stackrel{\Xi}{\leftrightarrows}$ & (n) & $\stackrel{\text { pq }}{m}$ & $\stackrel{0}{0}$ & 志 & $\stackrel{n}{n}$ & $\underset{\substack{\text { i } \\
i}}{ }$ & : & ષ્ণి & Эे & $\bar{\Xi}$ & $\overline{\mathrm{g}}$ & $\stackrel{i}{i}$ & స్․ & $\hat{o}_{i}^{\infty}$ & 安 & $\bar{m}$ & $\underset{+}{+}$ & है. & $\stackrel{\stackrel{\otimes}{े}}{i}$ \\
\hline$\cong$ & $\cong$ & \&̊. & $\frac{\infty}{m}$ & $\stackrel{\Xi}{\Xi}$ & $\tilde{o}_{i}$ & ले & हे & $\stackrel{+}{n}$ & 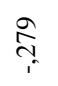 & $\tilde{8}$ & đ̇ & $\overrightarrow{\widehat{\sigma}}$ & $\stackrel{\tilde{N}}{\stackrel{1}{i}}$ & $\stackrel{ \pm}{\sigma}$ & $\overrightarrow{8}$ & $\stackrel{\infty}{\approx}$ & 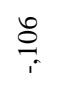 & ণ্ণ & $\vec{\delta}$ & $\hat{\varrho}$ & $\stackrel{0}{\dddot{n}}$ & ָे & 8 \\
\hline
\end{tabular}




\begin{tabular}{|c|c|c|c|c|c|c|c|c|c|c|c|c|c|c|c|c|c|c|c|c|c|c|c|}
\hline$\stackrel{\mathscr{g}}{\exists}$ & $\stackrel{\infty}{8}$ & $\underset{i}{\stackrel{1}{0}}$ & $\ddot{\&}$ & $\underset{i}{\stackrel{0}{0}}$ & ర్ర. & $\stackrel{0}{m}$ & $\stackrel{\infty}{\infty}$ & $\stackrel{\hat{s}}{\hat{i}_{i}}$ & స్ & 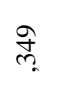 & $\stackrel{\hat{o}_{i}}{\hat{p}^{\prime}}$ & ठิ & $\widehat{\infty}$ & ల్ర. & $\stackrel{m}{\Rightarrow}$ & $\stackrel{8}{0}$ & $\stackrel{\Delta}{0}$ & 范 & 8 & $\underset{i}{\stackrel{\Xi}{\rightleftarrows}}$ & $\widetilde{o}_{i}$ & 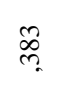 & $\underset{i}{+}$ \\
\hline$\stackrel{\Xi}{\Xi}$ & $\tilde{\varepsilon}$ & $\stackrel{\infty}{0}$ & $\stackrel{\infty}{0}_{i}^{\circ}$ & $\vec{n}$ & $\frac{\tilde{\infty}}{i}$ & $\stackrel{\curvearrowleft}{\leftrightharpoons}$ & $\stackrel{\mathscr{O}}{0}$ & : & $\overline{\tilde{\theta}}_{i}$ & $\underset{\text { 亲 }}{i}$ & 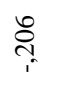 & $\stackrel{\overbrace{}}{\stackrel{r}{i}}$ & $\stackrel{\stackrel{\circ}{\circ}}{\stackrel{r}{\circ}}$ & $\stackrel{\infty}{\stackrel{\infty}{ }}$ & $\begin{array}{l}+ \\
\stackrel{D}{0} \\
i_{i}\end{array}$ & $\underset{i}{g}$ & $\stackrel{巳}{\leftrightharpoons}$ & 임 & ర్ & $\stackrel{0}{0}_{i}^{\infty}$ & $\overline{\bar{y}}$ & $\stackrel{\infty}{5}$ & సై \\
\hline$\cong$ & $\frac{8}{:}$ & J & $\stackrel{ \pm}{\varrho}$ & ठิ & छे & $\stackrel{\gtrless}{\stackrel{0}{0}}$ & $\stackrel{\mathbb{I}}{i}$ & $\stackrel{\tilde{\sim}}{i}$ & $\overline{8}$ & $\stackrel{\infty}{\simeq}$ & ठ̊. & $\stackrel{t}{0}_{i}$ & $\frac{a}{0}$ & $\frac{g}{i}$ & $\underset{\infty}{\infty}$ & $\underset{i^{r}}{g}$ & $\stackrel{\varrho}{\leftrightharpoons}$ & 오 & $\widetilde{\mathscr{\delta}}$ & $\stackrel{n}{?}$ & $\overrightarrow{0}$ & $\stackrel{\sim}{0}$ & $\begin{array}{l}\widehat{0} \\
i \\
1\end{array}$ \\
\hline$\stackrel{\circ}{=}$ & 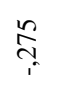 & $\tilde{\delta}$ & $\underset{\stackrel{+}{0}}{\stackrel{0}{0}}$ & $\stackrel{n}{\stackrel{n}{i}}$ & $\vec{\delta}$ & 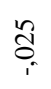 & $\hat{o}$ & $\underset{i}{+}$ & $\underset{\text { I }}{\stackrel{0}{0}}$ & $\stackrel{n}{\stackrel{n}{r}}$ & $\stackrel{\infty}{\mathbb{i}_{i}}$ & $\frac{\infty}{m_{1}}$ & $\stackrel{t}{\circ}$ & $\stackrel{\infty}{\stackrel{\infty}{\dddot{1}}}$ & $\stackrel{\infty}{\stackrel{\infty}{i}}$ & ळ. & $\stackrel{I}{I}$ & $\stackrel{\infty}{\dddot{T}}$ & $\stackrel{8}{i}$ & $\underset{I}{I}$ & 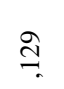 & $\stackrel{n}{\stackrel{n}{*}}$ & $\stackrel{\infty}{=}$ \\
\hline$气$ & $\tilde{c}_{i}$ & $\underset{i}{\stackrel{+}{0}}$ & $\underset{1}{\cong}$ & $\stackrel{\infty}{8}$ & $\vec{I}$ & 8 & $\stackrel{8}{\circ}$ & 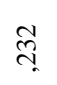 & $\stackrel{\because}{\dddot{I}}$ & $\stackrel{\infty}{\&}$ & 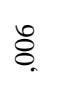 & $\tilde{\overbrace{}}$ & $\stackrel{\Delta}{\partial}$ & 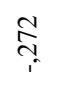 & $\underset{i}{\stackrel{\Delta}{*}}$ & 8 & స్రీ & $\tilde{s}_{i}^{\infty}$ & $\stackrel{ \pm}{\leftrightarrows}$ & 8 & $\Xi$ & $\frac{\Delta}{\sigma}$ & $\stackrel{0}{ \pm}$ \\
\hline$\stackrel{\infty}{=}$ & 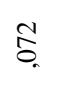 & $\underset{i}{\cong}$ & $\stackrel{8}{8}$ & $\begin{array}{l}0 \\
\stackrel{1}{1}\end{array}$ & Эิ & $\Xi$ & $\overline{8}$ & $\underset{i}{i}$ & o̊. & 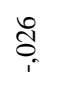 & $\mathbb{g}_{i}^{+}$ & i্ণ & $\stackrel{\infty}{\stackrel{\infty}{i}}$ & 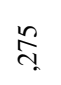 & 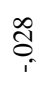 & $\overrightarrow{\sigma_{i}}$ & $\tilde{\delta}$ & है & 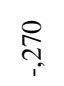 & $\stackrel{8}{\circ}$ & $\overline{\sigma_{i}}$ & $\overline{8}$ & $\mathbb{8}_{i}$ \\
\hline$\stackrel{\partial}{ }$ & $\stackrel{ \pm}{\stackrel{0}{0}}$ & $\stackrel{0}{\circ}$ & $\frac{\vec{\infty}}{i}$ & ర్రి. & $\stackrel{0}{ \pm}$ & $\tilde{\delta}$ & $\bar{s}$ & $\stackrel{\vartheta}{\stackrel{0}{i}}$ & 范 & 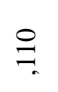 & $\stackrel{0}{0}$ & $\stackrel{+}{\oplus}$ & $\begin{array}{l}\infty \\
\stackrel{0}{i}\end{array}$ & $\stackrel{\infty}{\stackrel{\infty}{p}}$ & $\stackrel{ \pm}{O}$ & $\tilde{o}_{i}$ & $\underset{i}{\stackrel{\Xi}{\rightleftarrows}}$ & 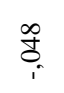 & $\underset{\infty}{\stackrel{n}{\infty}}$ & $\begin{array}{l}\infty \\
\infty_{i}^{\infty} \\
i,\end{array}$ & ले & $\frac{0}{m}$ & $\tilde{8}$ \\
\hline తి & $\cong$ & $\frac{\infty}{m}$ & ธి. & 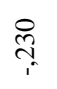 & $\ddot{B}_{i}$ & $\stackrel{\text { s}}{i}$ & 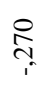 & $\stackrel{\infty}{\stackrel{+}{i}}$ & ळे & $\stackrel{\infty}{\stackrel{\infty}{0}}$ & ช్ & $\underset{m}{\stackrel{m}{r}}$ & $\frac{0}{2}$ & $\tilde{\delta}$ & $\stackrel{\sim}{\stackrel{\sim}{i}}$ & $\stackrel{\infty}{\infty}_{i_{1}}$ & $\stackrel{n}{n}$ & $\stackrel{\infty}{8}$ & 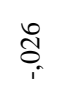 & : & 菅 & $\stackrel{\simeq}{0}$ & $\stackrel{\widetilde{\infty}}{\stackrel{\infty}{\sim}}$ \\
\hline $\bar{\beth}$ & $\stackrel{8}{\stackrel{\circ}{i}}$ & $\stackrel{ \pm}{=}$ & 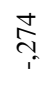 & סे. & $\tilde{g}_{i}$ & तે & $\stackrel{\Theta}{\simeq}$ & $\vec{I}$ & $\overline{\mathrm{g}}_{i}$ & $\stackrel{\Xi}{~}_{i}$ & $\underset{\sim}{\stackrel{+}{\sim}}$ & $\frac{\stackrel{0}{\circ}}{i}$ & $\frac{g}{i}$ & $\bar{s}$ & $\stackrel{\simeq}{m}$ & $\prod_{i}^{\infty}$ & 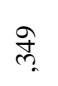 & 范 & $\underset{+}{+}$ & $\stackrel{n}{n}$ & $\tilde{\sigma}$ & $\stackrel{a}{0}_{i}$ & 8 \\
\hline$\tilde{\Xi}$ & 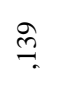 & $\check{g}_{i}$ & 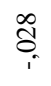 & $\vec{n}$ & 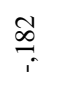 & $\stackrel{\varrho}{ٍ}$ & $\stackrel{\infty}{\stackrel{\infty}{0}}$ & $\underset{\text { ò }}{\text { i. }}$ & $\underset{i}{\stackrel{ \pm}{*}}$ & $\underset{i}{\stackrel{ \pm}{*}}$ & $\bar{\sigma}_{i}$ & $\underset{i}{\stackrel{f}{i}}$ & : & $\stackrel{\Re}{\stackrel{0}{r}}$ & $\vec{\partial}_{i}$ & $\stackrel{\mathscr{O}}{i}$ & $\stackrel{\vec{o}}{\hat{p}_{i}}$ & $\stackrel{\leftrightarrow}{\stackrel{\leftrightarrow}{1}}$ & हे & $\stackrel{\leftrightarrow}{i}$ & ণ্ণ & ô & 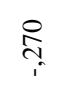 \\
\hline$\cong$ & $\begin{array}{l}\infty \\
\stackrel{0}{i}\end{array}$ & ले & $\vec{I}$ & $\stackrel{8}{0}$ & $\stackrel{8}{\mathscr{1}}$ & 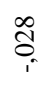 & $\stackrel{0}{\circ}$. & $\underset{i}{\stackrel{f}{*}}$ & $\stackrel{n}{\sim}$ & $\frac{0}{0}$ & $\overline{\mathrm{g}}$. & $\stackrel{\beth}{\beth}$ & $\stackrel{\circ}{=}$ & $\stackrel{n}{m}$ & $\tilde{c}_{i}$ & $\stackrel{+}{\simeq}$ & $\stackrel{\infty}{\stackrel{\infty}{\leftrightarrows}}$ & 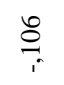 & $\stackrel{0}{\circ}$ & $\stackrel{8}{:}$ & तु & $\stackrel{m}{o}_{i}^{n}$ & e \\
\hline
\end{tabular}

\subsection{Construct Validity of the Measurement Tool (Explanatory Factor Analysis)}

The suitability of the data for analysis and sampling adequacy was determined by utilizing the Kaiser-Meyer-Olkin (KMO) test. The result of our KMO test is .836 and this value shows that the magnitude of the sample can be characterized as "excellent" for factor analysis and sample adequacy is very high (Kalaycı, 2010 Şencan, 2005; Tavşancıl, 2006). On the other hand, the results of Bartlett's test indicate that the chi square value $\left(X^{2}=6325.880(p<.01)\right.$ was significant. In conclusion, the correlation between variables is high. The test results are presented in Table 4 .

Table 4. Kaiser-Meyer-Olkin and Bartlett's Test Results

\begin{tabular}{lll}
\hline Kaiser-Meyer-Olkin Measure of Sampling Adequacy & 0,836 \\
\hline Bartlett's Test of Sphericity & Approx. Chi-Square & 6325.880 \\
& Degrees of freedom(df) & 199 \\
& Sig.(p) &, 000 \\
\hline
\end{tabular}

"The significance level is taken as $\mathrm{p}<0.01$

The Varimax rotation technique was performed and items with factor loadings lower than .40, items that load on more than one factor and small items with factor loadings less than 0.10 were extracted from the scale. Bütüner and Gür (2007), Yavuz (2005), proposed that scale items should not be loaded on more than one factor, the criteria for ideal value regarding the difference between the factor loadings should be at least 0.10 and items with factor loadings less than 0.10 should be called as similar items. 
Table 5. Factor Loadings of the Competency Scale for Lesson Planning

\begin{tabular}{lll}
\hline ITEMS & Factors & Factors \\
& 1 & 2 \\
\hline Item 3 & .712 & \\
Item 4 & .696 & \\
Item 5 & .675 & \\
Item 6 & .633 & \\
Item 7 & .587 & \\
Item 13 & .555 & \\
Item 16 & .522 & \\
Item 18 & .503 & \\
Item 22 & .497 & .788 \\
Item 23 & .466 & .756 \\
Item 1 & .442 & .711 \\
Item 2 & & .657 \\
Item8 & & .623 \\
Item 9 & & .602 \\
Item 10 & & .574 \\
Item 11 & & .551 \\
Item 12 & & .522 \\
Item 14 & & .498 \\
Item 15 & & .472 \\
Item 17 & & .466 \\
Item 19 & & \\
Item 20 & & \\
Item 21 & & \\
\hline the absom & & \\
\hline
\end{tabular}

As the absolute value below was determined as 0.40 , values less than .40 was suppressed in items sorted by descending. For this reason, factor loadings given in Table 5 refer to only those factor loadings more than 0.40 (Can, 2014). Factor loadings were determined as 0.40 to make scale items more qualified and distinctive.

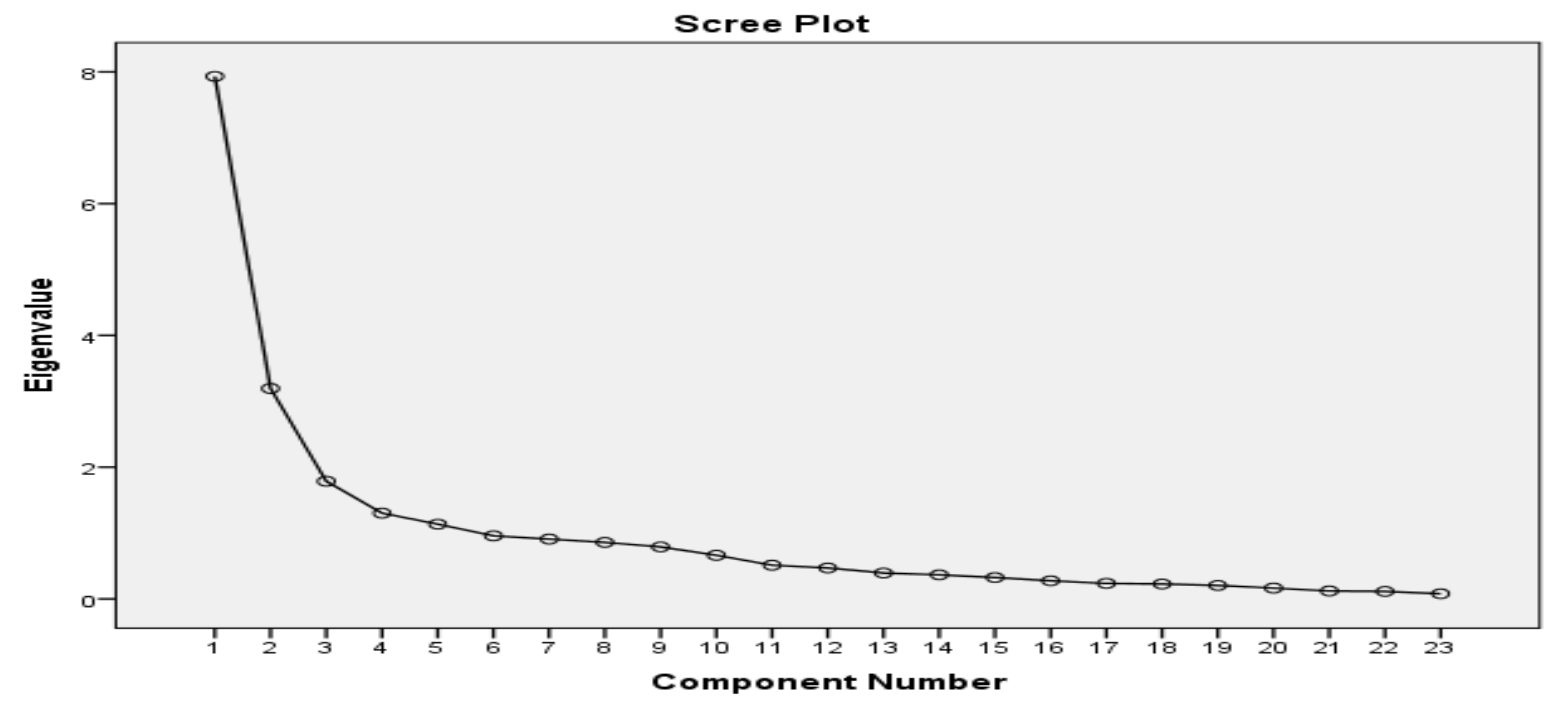

Figure 1. Line Graph for Eigenvalues

As seen from the Figure 1. Line Graph for Eigenvalues, the scale comprised of 2 factors. Considering the rapid decline following the first factor, we can contend that the scale has a general factor. Given that the first factor explains $34.489 \%$ of the total variance, this result was confirmed once again. On the other hand, the graphic yielded a horizontal shape after the second factor and did not show a downward sloping. To conclude, the scale has two-factor structure. Besides, looking at Table 6, we can understand that factors with eigenvalue greater than 1.00 were taken into account to identify the numbers of factors. 
Table 6. Factor Eigenvalues of the Competency Scale for Lesson Planning

\begin{tabular}{|c|c|c|c|c|c|c|c|c|c|}
\hline \multirow[b]{2}{*}{ 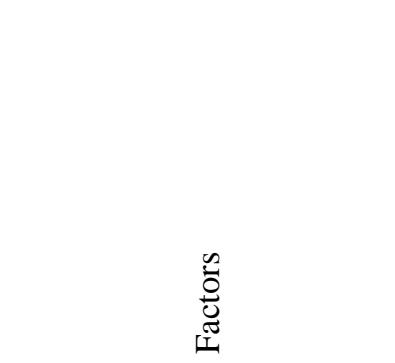 } & \multicolumn{3}{|c|}{ (Initial Eigenvalues) } & \multicolumn{3}{|c|}{$\begin{array}{l}\text { (Extraction Sums of } \\
\text { Squared Loadings) }\end{array}$} & \multicolumn{3}{|c|}{$\begin{array}{l}\text { Descriptive } \\
\text { Statistics }\end{array}$} \\
\hline & $\stackrel{\pi}{0}$ & 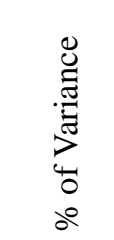 & $\begin{array}{l}\frac{\partial}{0} \\
\stackrel{D}{\Xi} \\
\frac{\vec{\Xi}}{\Xi} \\
\Xi \\
\Xi\end{array}$ & $\stackrel{\pi}{0}$ & 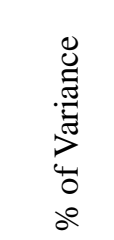 & 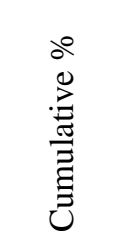 & 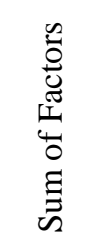 & 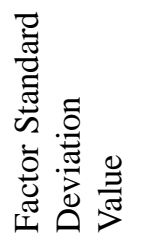 & 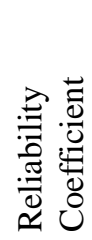 \\
\hline 1.Theoretical Competency & 3.687 & 47.658 & 47.658 & 9.687 & 47.658 & 47.658 & 61.68 & 7.254 & ,778 \\
\hline 2.Practical Competency & 1,252 & 17,958 & 65.616 & 5,252 & 17,958 & 65.616 & 31.12 & 4.325 & ,792 \\
\hline
\end{tabular}

The findings obtained from the factor analysis highlighted the presence of two factors with eigenvalues greater than 1. Therefore, we can determine "The Competency Scale for Lesson Planning" as a two-factor scale. Eigenvalues of these two factors and their explained variances were shown in Table 6. The factors were named as follows: "theoretical competency" (11 items) and "practical competency" (12 items). The eigenvalues of these factors, respectively, are 3.687 and 1.252 and accordingly the explanatory factor analysis indicated that these factors explained $47.658 \%$ and $17.958 \%$ of the Competency Scale for Lesson Planning, respectively.

The explanatory factor analysis (EFA) results revealed that these extracted two factors explained $65.616 \%$ of the total variance. SSencan (2005) and Can (2014) argued that this variance rate is acceptable. Pearson correlation coefficients were calculated to investigate the relation of the two factors between each other and with the total scale score and the results are shown in table 7. Based on the findings presented in Table 7, we see that the relation of the two factors between each other and with the total scale score was found significant. Depending on the correlation coefficients of the scale, its reliability is characterized as follows: if it ranges between $0.70-1.00$, the reliability of the scale is highly reliable; if it ranges between $0.69-0.30$, the reliability of the scale is moderately reliable; if it ranges between $0.29-0.00$, the reliability is low (Büyüköztürk, 2006).

Table 7. Correlation of the two factors with each other and the total scale

\begin{tabular}{llll}
\hline Factors & Factor 1 & Factor 2 & Total \\
\hline Theoretical competency (F1) & $*$ & & \\
Practical competency (F2) & .758 & $*$ & $*$ \\
Total & .863 & .833 & $*$ \\
\hline
\end{tabular}

*All correlations are taken as $\mathrm{p}<0.01$

According to the correlation analysis of two factors with each other and total scale, the correlation coefficients between total score and each factors were determined as follows: "theoretical competency" (factor 1 ) sub-dimension is $\mathrm{r}=.863$; "practical competency" (factor 2) sub-dimension is $r=.833$. Given that the relation between the two factors in the scale and total scale is highly significant, this result supports the construct validity of the Competency Scale for Lesson Planning. The results of the KMO and Bartlett's tests were supported as well.

\subsection{Language Validity of the Competency Scale for Lesson Planning}

The Competency Scale for Lesson Planning is 5-likert scale that consists of 23 items and 2 sub-dimensions. In this context, theoretical competency sub dimension consist of 11 items and practical competency sub dimension consist of 12 items. The scale was adapted to English language by two-people team. Afterwards, three out of six-people group majored in English Literature and Language was asked to translate English items to Turkish and the rest of the group were asked to translate Turkish items to English. As a result of the findings obtained, the scale was finalized in English. Then, English version of the scale was administrated to 35 students majoring in English Teaching. After 7 days passed, the Turkish version of the scale was carried out and the relationship between two versions was compared. In light of the data obtained, significance level was determined using Pearson's Product Moment Correlation Coefficient test and the significance level was calculated as .714. 
Table 8. Explanatory Factor Analysis

\begin{tabular}{lll}
\hline Fit Indices & Fit Range & $\begin{array}{l}\text { Research Model } \\
\text { Four-Factors Model }\end{array}$ \\
\hline Total Fit Index & \\
$\chi^{2} / s d$ & $0 \leq \chi^{2} / s d \leq 3$ & $527.35 / 199=2.65$ \\
Comparative Fit Index & & \\
NFI & $.90 \geq-\geq .94$ & .91 \\
NNFI & $.90 \geq-\geq .94$ & .92 \\
IFI & $.90 \geq-\geq .94$ & .93 \\
CFI & $\geq 95$ & .95 \\
RMSEA & $0.05 \leq-\leq 0.08$ & 0.068 \\
Absolute Fit Indices & $\geq .90$ & .92 \\
GFI & $\geq .85$ & .86 \\
AGFI & & \\
Residual Based Indexes & of & .063 \\
Compliance & & .077 \\
SRMR & $.06 \leq-\leq .08$ & \\
RMR & & \\
\hline
\end{tabular}

As seen in Table 8, a confirmatory analysis was performed to test the reliability of the two sub-dimensions identified through explanatory factor analysis. The results of CFA indicated that chi-square was $\left(\chi^{2}=527.35\right)$, degree of freedom $(\mathrm{df}=199, \mathrm{p}=0.00)$ was $\chi^{2} / \mathrm{df}=2.65 ; \mathrm{SRMR}=.063, \mathrm{RMR}=.077 ; \mathrm{AGFI}=.86 ; \mathrm{GFI}=.92 ; \mathrm{RMSEA}=0,068, \mathrm{CFI}=.95$, $\mathrm{NNFI}=.92, \mathrm{NFI}=.91, \mathrm{IFI}=.93$. CFA revealed that $\chi 2 / \mathrm{df}$ ratio is lower than 3 . Other goodness for fit indices computed by CFA was: $\mathrm{IFI}=.90 \geq-\geq .94$, NFI $=.90 \geq-\geq .94$., NNFI $=.90 \geq-\geq .94, \mathrm{CFI}=\geq, 95$, RMSEA $=0.05 \leq-\leq 0.08$ and $\mathrm{GFI}=\geq .90 \mathrm{AGFI}=\geq .85$ and lastly SRMR and RMR $=.06 \leq-\leq .08$. Consequently, the values mentioned above indicate acceptable fit (Şimşek, 2007; Y1lmaz and Çelik, 2009).

\subsection{Pre-service Teachers' Competencies in Lesson Planning}

The second sub-problem of the research seeks to the following question 'What is the competency level of the pre-service teachers in lesson planning?' using the Competency Scale for Lesson Planning. Correspondingly, arithmetic mean and standard deviation values were tabulated in Table 9.

Table 9. The Competency Levels of the Sample Group in Lesson Planning

\begin{tabular}{llllllllll}
\hline No & $\mathrm{N}$ & $\mathrm{X}_{\text {ort }}$ & $\mathrm{Ss}$ & Frequency Level & $\mathrm{No}$ & $\mathrm{N}$ & $\mathrm{X}_{\text {ort }}$ & $\mathrm{Ss}$ & Frequency Level \\
\hline I 3 & 620 & 4.69 & .878 & Agree Strongly & I 8 & 620 & 4.11 & .683 & Agree \\
I 6 & 620 & 4.67 & .585 & Agree Strongly & I 12 & 620 & 4.08 & .603 & Agree \\
I 13 & 620 & 4.64 & .789 & Agree Strongly & I 20 & 620 & 4.00 & .669 & Agree \\
I 16 & 620 & 4.56 & .652 & Agree Strongly & I 19 & 620 & 3.92 & .793 & Agree \\
I 1 & 620 & 4.53 & .874 & Agree Strongly & I 17 & 620 & 3.81 & .766 & Agree \\
I 22 & 620 & 4.52 & .745 & Agree Strongly & I 11 & 620 & 3.78 & .489 & Agree \\
I 4 & 620 & 4.50 & .653 & Agree Strongly & I 15 & 620 & 3.76 & .610 & Agree \\
I 23 & 620 & 4.44 & .604 & Agree Strongly & I 9 & 620 & 3.76 & .558 & Agree \\
I 1 & 620 & 4.42 & .701 & Agree Strongly & I 21 & 620 & 3.71 & .781 & Agree \\
I 7 & 620 & 4.33 & .556 & Agree Strongly & I 10 & 620 & 3.68 & .668 & Agree \\
I 5 & 620 & 4.30 & .457 & Agree Strongly & I 14 & 620 & 3.67 & .853 & Agree \\
I 2 & 620 & 4.27 & .417 & Agree Strongly & & & & & \\
A
\end{tabular}

An inspection of the data in the table 9 reveals that the lowest mean value is "item 3" $\left(\mathrm{X}_{\text {ort }}=4.69\right)$, and the highest is "item 14 " $\left(\mathrm{X}_{\text {ort }}=3.67\right)$ in the 23 item-scale. It is also understood that, arithmetic mean of the participation levels in the opinions on the first 12 items is "Strongly agree" and "Agree" for the remaining 11 items. The arithmetic average of all items is at "Agree" level $\left(\mathrm{X}_{\text {ort }}=4.18\right)$. The most striking result of the second sub-problem of the research is that the first 11 items measure the sub-dimension of theoretical competency.

It is seen that the mean rank of the items is distributed in a narrow range, namely, between 3.67 and 4.69 . It is thus observable that pre-service teachers' opinions on lesson planning competency are close to each other. To understand it more clearly, the graphic is illustrated in Figure 2. 


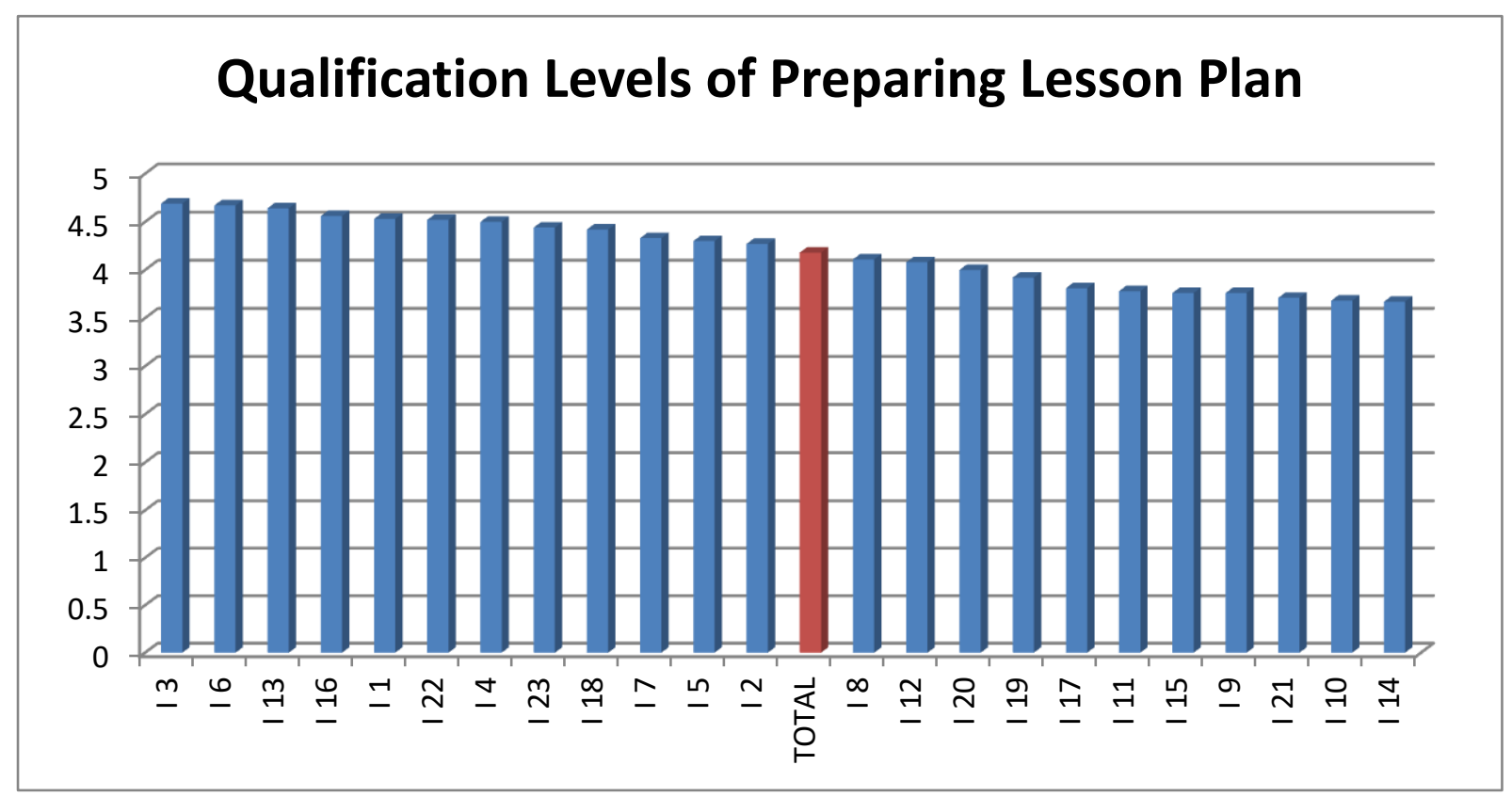

Figure 2. Competency Levels of Preparing Lesson Plan

3.5 The Significance Level of the Pre-service Teachers' Competencies in Lesson Planning with respect to the Variables Identified

The third sub-problem of the research seeks to the following question 'Do pre-service teachers' competency levels in lesson planning significantly vary according to gender, department, and grade level variables?". Accordingly, the normality test was applied to the research variables to find out how they were distributed.

Table 10. The Kolmogorov-Smirnov Test Administrated to the Variables Identified

\begin{tabular}{llll}
\hline Normality Test & \multicolumn{2}{l}{ Kolmogorov-Smirnov } & \\
& Statistic & Degree of Freedom & Level of Significance \\
Gender & .411 & 755 & .000 \\
Department & .305 & 755 & .000 \\
Grade Level & .286 & 755 & .000 \\
\hline
\end{tabular}

Kolmogorov-Smirnov (K-S) test is used to determine whether sample data is normally distributed. If the test indicates normality, parametric tests are performed, otherwise non-parametric tests are used. Non-parametric test is used when "p" value is significant at 0.05 . If the significance level is $\mathrm{p}<0.05$, then parametric test is employed (Can, 2014, p.89). Thus, Kolmogorov-Smirnov test was conducted and the significance level of the test was found .05 according to all variables identified. Then, non-parametric tests were utilized. Mann Whitney U test was first used to determine if the gender variable had a significant effect on the competency levels of the pre-service teachers in lesson planning.

Table 11. Significance Level of the Pre-Service Teachers' Competency Levels in Lesson Planning on the "Gender" Variable

\begin{tabular}{llllllll}
\hline & Gender & $\mathrm{N}$ & Mean Ranks & Sum Total & $\mathrm{U}$ & $\mathrm{Z}$ & $\mathrm{P}$ \\
\hline Theoretical & Female & 480 & 266.42 & 138741.5 & 13533.5 & $-1,205$ & $.014^{*}$ \\
Competency & Male & 140 & 260.61 & 110243.0 & & & \\
$\begin{array}{l}\text { Practical } \\
\text { Competency }\end{array}$ & Memale & 480 & 239.27 & 127749.5 & 10541.5 & $-1,254$ & $.000^{*}$ \\
\hline
\end{tabular}

*The significance level is taken as $\mathrm{p}<0.05$

Given the results of Mann Whitney U test, the gender variable leads to statistically significant differences in the two sub-dimensions. Comparing the mean rank scores between female and male students as to the sub-dimension of theoretical competency, it is found out that the mean rank of female pre-service teachers is 266.42 (U:13533; Z:-1.205) and the mean rank of male pre-service teachers is 260.61 (U: 13533; Z:-1.205). With this in mind, we can contend that female pre-service teachers have higher levels of theoretical competency in lesson planning than male pre-service teachers.

On the other hand, in terms of the sub-dimension of the practical competency, the result found is in favour of male pre-service teachers. From table 11, we can clearly see that the mean rank of male pre-service teachers is 243.00 (U: 
10541.5; Z: -1.254) and the mean rank of female pre-service teachers is 239.27 (U: 10541.5; Z: -1.254). This finding indicates that male pre-service teachers have higher levels of practical competency in lesson planning when compared to female pre-service teachers.

Table 12. The Significance Level of the Pre-service Teachers' Competency Levels in Lesson Planning Regarding the "Department" Variable

\begin{tabular}{llllll}
\hline & Departments & N & & df & Mean Ranks \\
& & & & & \\
Classroom Teaching & 138 & 229.93 & & \\
Theoretical & Preschool Teaching & 174 & 221.49 & $.037^{*}$ \\
Competency & Science Teaching & 65 & 219.73 & 3.272 & 4 \\
& Social Sciences Teaching & 100 & 224.01 & & \\
& Turkish Language Teaching & 70 & 202.96 & & \\
& Classroom Teaching & 138 & 213.74 & & .201 \\
Practical & Preschool Teaching & 174 & 249.49 & & \\
Competency & Sathematics Teaching & 65 & 255.40 & & \\
& Science Teaching & 100 & 241.67 & & \\
& Social Sciences Teaching & 70 & 248.78 & & \\
\hline
\end{tabular}

*The significance level is taken as $\mathrm{p}<0.05$

The second sub-problem of the research examined whether the department variable had a significant effect on pre-service teachers' competencies in lesson planning and the results were presented in Table 13. As a consequence, a significant level of .05 were detected between at least two groups $(=3.272$; df: $4 ; \mathrm{p}=.037)$ only as to the sub-dimension of theoretical competency. Afterwards, Dunnett-C analysis multi-comparison test was run to identify significant differences between the groups. Dunnett-C multiple comparison test (Post-Hoc), which can be used for non-parametric variables or when the variances are not equal, is based on average mean rank and q-distribution. (Gunlu, 2016).

Table 13. Dunnett - C Test for the "Department" Variable

\begin{tabular}{|c|c|c|c|c|c|c|}
\hline & 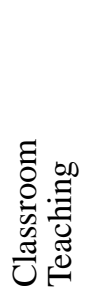 & 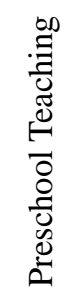 & 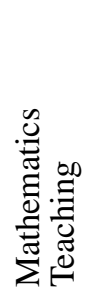 & 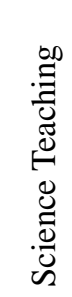 & 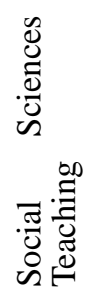 & 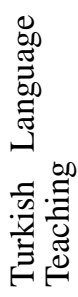 \\
\hline Classroom Teaching & & * & $*$ & * & * & * \\
\hline Preschool Teaching & * & & & & & \\
\hline Mathematics Teaching & $*$ & & & & & \\
\hline Science Teaching & * & & & & & \\
\hline Social Sciences Teaching & * & & & & & \\
\hline Turkish Language Teaching & * & & & & & \\
\hline
\end{tabular}

As a result of the Dunnet-C analysis, significant differences between the classroom teaching pre-service teachers and pre-services teachers majoring in all other departments in the sample group were found. Mann Whitney U test was employed to identify significant differences between classroom teaching group and other groups. The results are detailed in Table 14.

Table 14. Mann Whitney U Test Applied To the Groups within "Departments" Variables

\begin{tabular}{|c|c|c|c|c|c|c|}
\hline Departments & $\bar{N}$ & Mean Rank & Sum of Ranks & $\bar{U}$ & $\mathrm{Z}$ & $\mathrm{p}$ \\
\hline Classroom Teaching & 138 & 248.05 & 2779.5 & 525500 & -1.145 & $.005^{*}$ \\
\hline Preschool Teaching & 174 & 241.56 & 2566.5 & & & \\
\hline Classroom Teaching & 138 & 236.65 & 2689.5 & 469.200 & -1.587 & $.002^{*}$ \\
\hline $\begin{array}{l}\text { Mathematics Teaching } \\
\text { Classroom Teaching }\end{array}$ & $\begin{array}{l}65 \\
138\end{array}$ & $\begin{array}{l}234.14 \\
239.77\end{array}$ & $\begin{array}{l}2599.0 \\
2698.4\end{array}$ & & & \\
\hline Science Teaching & $\begin{array}{l}150 \\
100\end{array}$ & 236.41 & $\begin{array}{l}2098.4 \\
2100.5\end{array}$ & 488.630 & -1.263 & $.012^{*}$ \\
\hline Classroom Teaching & 138 & 240.78 & 2564.7 & 500.474 & -.1 .006 & $.009^{*}$ \\
\hline $\begin{array}{l}\text { Social Science Teaching } \\
\text { Classroom Teaching }\end{array}$ & $\begin{array}{l}70 \\
138\end{array}$ & $\begin{array}{l}235.55 \\
241.66\end{array}$ & $\begin{array}{l}2690.3 \\
21450\end{array}$ & & & \\
\hline Turkish Language Teaching & 73 & 237.98 & 2296.0 & 511.648 & -1.638 & $.042^{*}$ \\
\hline
\end{tabular}

${ }^{*}$ The significance level is taken as $\mathrm{p}<0.05$ 
The research findings lead us to a striking result. As known, Mann Whitney U test was applied to all departments. As shown in table 14, mean ranks of the all items obtained through Mann Whitney $\mathrm{U}$ analysis yielded significant difference in favour of classroom teaching group. We can thus contend that classroom teaching pre-service teachers demonstrate higher levels of theoretical competency in lesson planning than other pre-service teachers from other departments.

Table 15. The Significance Level of the Pre-service Teachers' Competency Levels in Lesson Planning Regarding the "Grade" Variable

\begin{tabular}{|c|c|c|c|c|c|c|c|}
\hline & Grade & $\mathrm{N}$ & Mean Rank & Sum Of Ranks & $\mathrm{U}$ & $\mathrm{Z}$ & $\mathrm{P}$ \\
\hline Theoretical & 3rd Grade & 375 & 301.45 & 178691.5 & 11253.5 & $-1,669$ & $.011^{*}$ \\
\hline Competency & 4th Grade & 245 & 307.69 & 160128.0 & & $-1,007$ & \\
\hline $\begin{array}{l}\text { Practical } \\
\text { Competency }\end{array}$ & $\begin{array}{l}\text { 3rd Grade } \\
\text { 4th Grade }\end{array}$ & $\begin{array}{l}375 \\
245\end{array}$ & $\begin{array}{l}298.58 \\
306.57\end{array}$ & $\begin{array}{l}15699.5 \\
1135110\end{array}$ & 13651.5 & $-1,754$ & $.001^{*}$ \\
\hline
\end{tabular}

"The significance level is taken as $\mathrm{p}<0.05$

Given the data tabulated in Table 15, we see significant differences between two sub-dimensions. Concordantly, the mean rank of senior pre-service teachers in the sub-dimension of theoretical competency was calculated as 307.69 (U:11253.5; Z:-1.669), whereas the mean rank of 3rd grade pre-service teachers was calculated as 301.45 (U:11253.5; Z:-1.669). Similarly, the mean rank of senior pre-service teachers in the sub-dimension of practical competency was calculated as 306.57 (U:13651.5; Z:-1.754), whereas the mean rank of 3rd grade pre-service teachers was calculated as 298.58 (U: 13651.5; Z:-1. 1.754). Consequently, we can interpret that senior pre-service teachers have higher levels of theoretical and practical competency in lesson planning than $3^{\text {rd }}$ grade pre-service teachers.

\section{Discussion and Suggestions}

This study sought to identify the competency levels of 3rd year and senior pre-service teachers from the Faculty of Education in preparing a lesson plan. According to the regulations of the Ministry of National Education, teachers are responsible for preparing a daily lesson plan in the 2018-2019 academic year. For that reason, the present study is of vital importance. In light of the observations made and expert opinions of faculty members teaching lesson planning, the study revealed that pre-service teachers' competency level in lesson planning were not satisfactory. Also, public and private sector teachers' responses to open-ended questions demonstrated that teachers' theoretical and practical skills in lesson planning were not reliable. In this respect, the competency scale for lesson planning was developed to identify both pre-service teachers' and teachers' weaknesses in lesson planning and to contribute to the literature and future researches. Previous researches denoted that preparing lesson plan will highly contribute to teachers' instructional process and academic achievement (Zahorik 1970, Freiberg and Driscoll, 1992; Küçükahmet, 1999; Bilen 2002; Ercoşkun, Nalçacı, Kılıç, 2004; Kara and Koca, 2004; Demirel, 2006; Haşlaman, Mumcu, Uslue, 2010; Kablan, 2012).

When it comes to the sub-problem, mean ranks of the scale were evaluated and the competency levels of the pre-service teachers in lesson planning were analyzed. Correspondingly, mean scores were categorized from the highest to the lowest. The most striking result thus is that the first 11 items were related with theoretical competency sub-dimension, whereas the remaining 12 items were related with practical competency sub-dimension. We therefore can argue that pre-service teachers perceive themselves competent in lesson planning in terms of theory. To put it differently, we can interpret that pre-service teachers can easily prepare a lesson plan in theory when asked to do it, while they barely apply a lesson plan in practice. Bearing in mind that Senemoglu (2005) highlights through a planned instructional process, teachers will feel self-confident and easily handle unexpected occasions in the classroom environment by behaving calm and easy, creating environments where pre-service teachers can realize their planned instructional program will highly contribute to raise more qualified teachers.

The literature review indicates that few studies have examined teachers or pre-service teachers' competencies in lesson planning, which increases the importance of the study. For that reason, the current study attempted to identify whether service teachers' competencies in lesson planning significantly differentiate by various variables so that an in-depth appreciation of the study was targeted. Thus, gender, department and grade level variables were included and a series of tests were conducted. As a result, it was found out that these three variables had some effects on pre-service teachers' competencies in lesson planning.

Looking at gender variable, we can see that female students are more competent than male students with respect to the sub-dimension of theoretical competency. However, male students were more competent than female students in practicing lesson plan. A close attention should be paid to this finding. In particular, faculty members who deliver lesson planning and implementation courses should monitor pre-service teachers more carefully and help both female and male students overcome their weakness in theory and practice so that a balance between female and male students can be achieved. In this sense, the causes of this outcome should be examined meticulously.

In terms of the grade level variable, it was detected that senior pre-service teachers had higher levels of competency in the sub-dimensions of theoretical and practical Competency when compared to 3rd grade pre-service teachers. The underlying reason of it was that senior pre-service teachers had been taught teaching practice. Additionally, teaching practice course 
involves gains regarding the lesson plan preparation and implementation and pre-service teachers experience actual teaching in actual classroom environment which clearly explained why senior pre-service teacher had higher levels of competency. Baum and King (2006) assert that high quality teaching practice will foster collaborations between faculties and the schools, encourage cooperation and active learning among students, improve active learning, yield helpful feedbacks, equip with time management skills, increase their expectations and enable students to explore different ways of learning. A number of other studies also reveal that teaching practice courses provide a positive contribution to pre-service teachers' lesson planning skills (Kiraz 2002; Karamustafaoğlu and Akdeniz, 2002; Azar, 2003; Şişman and Acat, 2003; Özbek and Aytekin,2003; Hascher, Cocard and Moser, 2004; Dallmer, 2004; Gökçe and Demirhan, 2005).

According to Bolat (2007) and Süral \& Dedebali (2018), pre-service teachers' knowledge and skills on curriculum literacy should be identified so that they adopt a curriculum-based approach for their class activities. In this sense, curriculum literacy plays a significant role in educating pre-service teachers and improving their competency in teaching. That is to say, pre-service teachers should be equipped with curriculum components. Therefore, they can develop enhanced literacy skills. According to Konyalıŏlu and Iş̧1k's experimental study (2003), the group who were taught with a lesson plan showed statistically higher success in learning when compared those who were not taught with a lesson plan. The mentioned study emphasized that lesson plan preparation increases the quality of teaching and positively contribute to students' academic achievements. Each and every pre-service teacher should know how to organize a lesson plan and has the awareness on the importance of the lesson planning. In a nutshell, individuals should be competent in preparing a lesson plan, in other words, their academic background should be education majors.

\section{References}

Azar, A. (2003). Reflections of views on school experience and teaching practice, Milli Eğitim Dergisi, 159.

Balc1, A. (1995). Research methods, techniques and principles in social sciences, Ankara: Pegem Yayıncilik.

Baum, A. C., \& King, M. A. (2006). Creating a climate of self-awareness in early childhood teacher preparation programs. Early Childhood Education Journal, 33(4), 217-222. https://doi.org/10.1007/s10643-005-0050-2

Beeth, M. E., \& Adadan, E. (2006). The influences of university-based coursework on field experience. Journal of Science Teacher Education, 17(2), 103-120. https://doi.org/10.1007/s10972-006-9013-8

Bilen, M. (2002). Teaching from Plan to Application. 6. Basım, Ankara:Anı Yayıncılık.

Bolat, Y. (2017). The concept of education program literacy and education program literacy scale. Electronic Turkish Studies, 12(18). https://doi.org/10.7827/TurkishStudies.12103

Bütüner, Ö. S., \& Gür, H. (2007). Developing an attitude scale for V diagram, Milli Eğitim Dergisi, 176 (1), 72-85.

Büyüköztürk, Ş. (2006). Data analysis for Social Sciences. Ankara: Pegem A Yayıncılık.

Can, A. (2014). Quantitative data analysis in scientific research process with SPSS. Ankara: Pegem A Yayıncllı.

Dallmer, D. (2004). Collaborative relationships in teacher education: A personal narrative of conflicting roles. Curriculum Inquiry, 34(1), 29-45. https://doi.org/10.1111/j.1467-873X.2004.00279.x

Demirel, Ö. (1999). The Art of Teaching from Planning to Evaluation. Ankara, Pegema Yayıncılık.

Driscoll, A., \& Freiberg, J. H. (1992). Universal teaching strategies.

Gökçe, E. (2005). Teacher Candidates and Supervising Teachers' Opinions about Activities of Teaching Practice in Elementary Schools. Ankara üniversitesi eğitim bilimleri fakültesi Dergisi, 38(1), 43-72.

Goodlad, J. (1991). Why we need a complete redesign of teacher education. Educational Leadership, 49, 4-6.

Günlü, Z. (2016). Comparing of Some Multiple Comparision Test in the Dam's Age and Enterprises Effects on Growth Rate of Hair Goat Kids, (yayımlanmamış yüksek lisans tezi), Selçuk Üniversitesi Sağlık Bilimleri Enstitüsü, Konya.

Hascher, T., Cocard, Y., \& Moser, P. (2004). Forget about theory-practice is all? Student teachers' learning in practicum. Teachers and teaching, 10(6), 623-637. https://doi.org/10.1080/1354060042000304800

Haşlaman, T., Mumcu, F. K., \& Usluel, Y. K. (2010). The Integration of Information and Communication Technologies in Learning and Teaching Process: A Lesson Plan Example. Eğitim ve Bilim, 32(146), 54-63.

Kablan, Z. (2012). The Effects of Level of Cognitive Learning and Concrete Experience on Teacher Candidates' Lesson Planning and Application Skills, Education and Science, 37(163), 239-253.

Kalaycı, Ş. (2010). Factor Analysis SPSS Applied Multivariate Statistical Techniques. (Edt: Ş. Kalaycı) Ankara: Asil Yayın Dağıtım.Kara, Y., \& Özgün-Koca, S. A. (2004). Learning through discovery and application of meaningful learning approaches in mathematics courses: Two lesson plans on "squaring the sum of two terms. Illkögretim online, 3(1). 
Karamustafaoğlu, O., \& Akdeniz, A. R. (2002). Ability to reflect the expected behavior of pre-service physics teachers in practice schools. V. Ulusal Fen Bilimleri ve Matematik Ĕ̈itimi Kongresi, 1, 456-769.

Kılıç, D., Nalçacı, A., \& Ercoşkun, H. (2004). Plans and problems encountered in primary education. XIII. Ulusal Eğitim Bilimleri Kurultayl, 6-9.

Kiraz, E. (2002). The function of practice teachers in pre-service professional development of pre-service teachers. Eğitim Bilimleri ve Uygulama, 1(2), 183-196.

Konyalığlu, A. C.; Konyalıŏlu, S., \& Işık, A. (2002). On Planned Education in Mathematics Courses. Kastamonu Eğitim Dergisi. 10(2), 351-358.

Küçükahmet, L. (2003). Planning and Evaluation in Teaching, Nobel Yayın, Ankara.

Meade, E. (1991). Reshaping the clinical phase of teacher preparation. Phi Delta Kappan, 72, 666- 669.

Millî Eğitim Bakanlığı (2018). Directive on the Implementation of Training and Training Activities. Tebliğler Dergisi, Ağustos 2018.

Özbek, T. Z., \& Aytekin, F. (2003). A research on views of prospective teachers about teaching profession and satisfaction of the students from teaching practice. Journal of Contemporary Education, 284, 31-39.

Peker, M. (2009). Opinions of prospective mathematics teachers about extended micro teaching experiences. Türk Eğitim Bilimleri Dergisi, 7(2), 353-376.

Roth, W. M., \& Tobin, K. (2001). Learning to teach science as practice. Teaching and Teacher Education, 17, 741-762. https://doi.org/10.1016/S0742-051X(01)00027-0

Sachs, J. (1997). Revisioning teacher education, Unicorn, 23, 46-56.

Şencan, H. (2005). Reliability and validity in social and behavioral measurements. Ankara: Seçkin Yayıncılık.

Senemoğlu, N. (2003). Development Learning and Teaching From Theory to Practice. Ankara, Gazi Kitabevi.

Senemoğlu, N. (2005). Development Learning and Teaching From Theory to Practice, Gazi Kitabevi, Ankara.

Şimşek, Ö. (2007). Development of Marmara learning styles scale and examination of learning styles of 9-11-year-old children. Yayınlanmamış Doktora Tezi. İstanbul: İstanbul Üniversitesi.

Şişman, M., \& Acat, M. B. (2003). The effect of teaching practice studies on the perception of teaching profession. Firat Üniversitesi Sosyal Bilimler Dergisi, 13(1), 235-250.

Sönmez, V. (2005). The Book of Curriculum Development. Ankara, Anı Yayıncılık.

Sönmez, V. (2017). Teaching Principles and Methods. Ankara, Anı Yayıncılık.

Sumpter, R. D. (1995). Expanding field experiences: Reality, results, and revision. Teacher Educator, 30, 6-15. https://doi.org/10.1080/08878739509555089

Sural, S., \& Dedebali, N. C. (2018). A Study of Curriculum Literacy and Information Literacy Levels of Teacher Candidates in Department of Social Sciences Education. European Journal of Educational Research, 7(2), 303-317.

Tan, Ş., Kayabaşı, Y., \& Erdoğan, A. (2002). Planning and Evaluation of Teaching, Anı Yayıncılık, Ankara.

Tavşancıl, E. (2006). Measurement of attitudes and data analysis with SPSS. (3. Bask1). Ankara: Nobel Yayınları.

Tigchelaar, A., \& Korthagen, F. (2004). Deepening the exchange of student teaching experiences: Implications for the pedagogy of teacher education of recent insights into teacher behaviour. Teaching and Teacher Education, 20(7), 665-679. https://doi.org/10.1016/j.tate.2004.07.008

Vural, B. (2006). Education - Planning in Education - Measurement and Strategies. İstanbul, Hayat Yayınları.

Yavuz, S. (2005), Developing a technology attitude scale for pre-service chemistry teachers, The Turkish Online Journal of Educational Technology, 4(1).

Yılmaz, V., \& Çelik, H. E. (2009). Structural Equation Modeling with LISREL. Ankara: Pegem Akademi; 53- 61.

Zahorik, J. A. (1970). The effect of planning on teaching. The Elementary School Journal,71(3), 143-151. https://doi.org/10.1086/460625

\section{Copyrights}

Copyright for this article is retained by the author(s), with first publication rights granted to the journal.

This is an open-access article distributed under the terms and conditions of the Creative Commons Attribution license which permits unrestricted use, distribution, and reproduction in any medium, provided the original work is properly cited. 